\title{
Mid-infrared long-pass filter for high-power applications based on grating diffraction
}

\author{
Daniel Gerz, ${ }^{1,2}$ Wolfgang Schweinberger, ${ }^{1,3}$ Thomas Patrick Butler, ${ }^{2}$ (1) Thomas Siefke, ${ }^{4,5}$ \\ Martin Heusinger, ${ }^{4}$ Tatiana Amotchinan, ${ }^{1}$ Vladimir Pervak, ${ }^{1}$ Uwe Zeitner, ${ }^{4,6}$ and loachim Pupeza ${ }^{1,2, *}$ (1) \\ ${ }^{1}$ Ludwig Maximilians University Munich, Am Coulombwall 1, 85748 Garching, Germany \\ ${ }^{2}$ Max Planck Institute of Quantum Optics, Hans-Kopfermann-Str. 1, 85748 Garching, Germany \\ ${ }^{3}$ King Saud University, Department of Physics and Astronomy, Riyadh 11451, Saudi Arabia \\ ${ }^{4}$ Institute of Applied Physics, Abbe Center of Photonics, Friedrich-Schiller Universität Jena, Albert-Einstein-Str. 15, 07745 Jena, Germany \\ ${ }^{5}$ Physikalisch-Technische Bundesanstalt, Bundesallee 100, 38116 Braunschweig, Germany \\ ${ }^{6}$ Fraunhofer Institute for Applied Optics and Precision Engineering, Albert-Einstein-Str. 7, 07745 Jena, Germany \\ ${ }^{*}$ Corresponding author: ioachim.pupeza@mpq.mpg.de
}

Received 25 January 2019; revised 7 May 2019; accepted 7 May 2019; posted 8 May 2019 (Doc. ID 358778); published 7 June 2019

\begin{abstract}
A gold-coated silicon grating has been developed, enabling efficient spatial separation of broadband mid-infrared (MIR) beams with wavelengths $>5 \mu \mathrm{m}$ from collinearly propagating, broadband, high-power light in the near-infrared (NIR) spectral range (centered at $2 \mu \mathrm{m}$ ). The optic provides spectral filtering at high powers in a thermally robust and chromaticdispersion-free manner such as that necessary for coherent MIR radiation sources based on parametric frequency downconversion of femtosecond NIR lasers. The suppression of a $>20 \mathrm{~W}$ average-power, $2 \mu \mathrm{m}$ driving pulse train by three orders of magnitude, while retaining high reflectivity of the broadband MIR beam, is presented. () 2019 Optical Society of America
\end{abstract}

https://doi.org/10.1364/OL.44.003014

Molecular spectroscopy in the mid-infrared spectral range, particularly in the "molecular fingerprint region" (500-4000 cm is a widely used analytical tool for applications ranging from fundamental science [1] to applied and life sciences [2], environmental monitoring, and quality control [3]. Crucial to the improvement of the sensitivity, specificity, and availability of such techniques is the development of robust, table-top sources of coherent mid-infrared (MIR) radiation. In recent years, the advent of high-power, near-infrared (NIR) femtosecond lasers has provided a way to reach the MIR regime through nonlinear optical methods. In particular, laser systems delivering trains of pulses with durations of a few tens of femtoseconds, multi$10 \mathrm{MHz}$ repetition rates, and several tens of watts of average power are available today, both at $1[4,5]$ and $2 \mu \mathrm{m}[6,7]$ central wavelengths. Via parametric frequency downconversion in suitable nonlinear crystals [8], these lasers have recently established a new class of MIR radiation sources [9-14], combining broad spectral coverage with brilliance values exceeding, even those of third-generation synchrotrons $[11,14]$, as well as with excellent temporal coherence $[15,16]$.
In nonlinear sources based on the processes of optical parametric amplification [10] or intrapulse difference frequency generation (IPDFG) [11-16], a common goal is to generate an isolated MIR idler beam, which can be used as a probing signal in a spectroscopic measurement. Therefore, this requires a method of separating the NIR driving pump/signal beam(s) from the generated MIR light. Typically, a dielectric-coated substrate is used in order to separate the NIR beam while transmitting the MIR wavelengths, with desirable features such as high separation contrast, steep slope, high transmission, and broad blocking range $[17,18]$. Employing chirped mirrors additionally allows for tailoring the pulse dispersion [19]. However, the production of these optics in the MIR spectral region remains challenging due to technical issues such as the peeling of layers owing to the internal stress of thick layers and the scarcity of suitable MIR transparent coating materials. In IPDFG, the use of femtosecond driving pulses can also result in relatively short $(<100 \mathrm{fs})$ and broadband idler pulses. In time-resolved applications such as pump-probe or electricfield-resolved spectroscopy [15], the temporal profile of the MIR field is essential and, therefore, any excess temporal broadening due to transmission through dispersive materials is disadvantageous. Furthermore, absorption of the dielectric substrate can limit the MIR bandwidth, as well as lead to thermal issues in the case of absorption of a driving beam of several tens of watts.

To address the challenge of dispersion-free, high-power filtering in parametric downconversion experiments, we have developed a novel diffraction-grating-based optic. The optic has been designed to suppress a nearly octave-wide spectrum centered at $2 \mu \mathrm{m}$ with a power of a few tens of watts, while retaining as much of the broadband MIR spectrum above $5 \mu \mathrm{m}$ as possible. In this Letter, we present the design and fabrication of the optic, as well as experimental characterization of the diffraction efficiency and proof-of-principle experiments showing the power scalability up to $40 \mathrm{~W}$ and suitability for current parametric MIR sources. 
A schematic of the operating principle of the long-pass filter is shown in Fig. 1(a). Two collinearly propagating beams are incident on the gold-coated silicon diffraction grating. The sub-MIR-wavelength reflecting structure efficiently diffracts the shorter wavelength NIR radiation into the 1st diffraction order (DO), while the MIR radiation is reflected specularly, remaining in the 0th DO. Similar approaches were previously employed in infrared monochromators utilizing echelette gratings in order to suppress shorter wavelength stray light [20]. More recently, beam splitting gratings have been used to separate extreme ultraviolet light from the driving NIR beam in high-harmonic generation [21,22]. The 0th DO corresponds mathematically to a specular reflection and, thus, is dispersionfree. In order to provide good thermal conductivity and facilitate production, the $30 \mathrm{~mm} \times 30 \mathrm{~mm}$ grating structure was imprinted into a silicon substrate using electron-beam lithography and reactive ion etching [23]. High reflectivity is ensured by coating the grating with a $140 \mathrm{~nm}$ gold layer $(3 \mathrm{~nm}$ of chromium used as an adhesive layer), deposited using an electronbeam-evaporation-based deposition plant (SYRUSpro 710). The grating period of $7 \mu \mathrm{m}$ leads to a diffraction angle of $16.5^{\circ}$ between the 0 th and the 1 st DO for a wavelength of $2 \mu \mathrm{m}$.

Simulations of the diffraction efficiency were carried out using the MATLAB-based grating diffraction calculation tool GD-Calc [24] with the optical constants of thin gold film on $\mathrm{SiO}_{2}$ found in Ref. [25]. This numerical tool uses a generalized variant of the rigorous coupled-wave diffraction theory. Several geometric parameters are used as inputs to the numerical model. For the light beams, the incident angle $\theta$ is defined as the angle between the propagation vector of the light and the normal vector to the grating plane, as shown in Fig. 1(a). The azimuthal angle $\varphi$ is the angle between the incoming light vector projected onto the grating plane and the grating vector $v_{g}$, shown in Fig. 1(a). A grating depth of $50 \%$, and grating depth of $600 \mathrm{~nm}$ were used. A high truncation order of 160 was chosen for the Fourier series in order to ensure a convergence with an error well below one percent relative diffraction

(a)

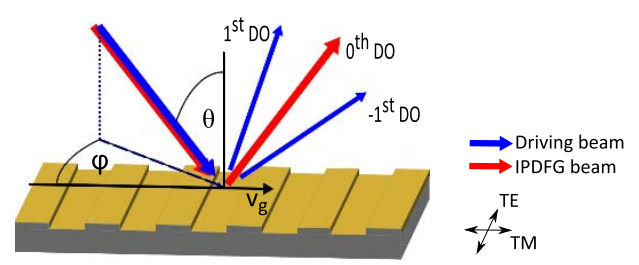

(b)

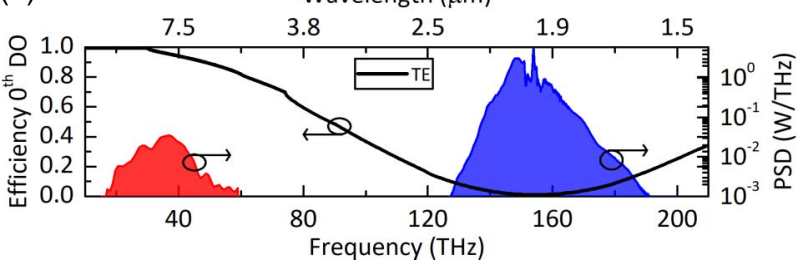

Fig. 1. Concept of a grating as a long-pass filter. (a) Binary grating diffracts the long wavelength beam mainly into 0th DO, while the short wavelength driving beam is mainly diffracted into higher DOs. $\theta$, incident angle; $v_{g}$, grating vector; TE, transverse-electric polarization; TM, transverse-magnetic polarization (b) Simulated diffraction efficiency into 0th DO for an IPDFG example (IPDFG beam, $\sim 0.3 \mathrm{~W}$; driving beam, $23 \mathrm{~W})$ [14]. efficiency. Figure 1(b) shows a calculated diffraction efficiency curve plotted together with the NIR and MIR spectra from Ref. [14]. The simulated diffraction efficiency shows a $>80 \%$ reflection into the 0th $\mathrm{DO}$ for wavelengths $>5 \mu \mathrm{m}$, with a minimum efficiency of $1 \%$ at $1.96 \mu \mathrm{m}$, chosen to coincide with the peak of the NIR spectrum.

The fabricated gratings were characterized using a Fouriertransform infrared (FTIR) spectrometer (Bruker, Vertex 70), together with an accessory for near-normal specular reflectance (Pike Technologies, 10Spec). The experimental setup of the FTIR characterization is shown in Fig. 2(a). A wire-grid polarizer was used to set the polarization of the incident field. Figure 2(b) presents the experimentally measured diffraction efficiencies for the TE- and TM-polarizations $\left(\theta=10^{\circ}\right.$; $\left.\varphi=0^{\circ}, 90^{\circ}\right)$, along with the numerically calculated efficiencies. Excellent agreement is observed between the measured data and numerical model for $\varphi=0^{\circ}$. Small deviations are found in the TM case, with the model predicting some sharp features near 5.8 and $8.3 \mu \mathrm{m}$ whose spectral position is very sensitive to the grating depth and the width of the ridge. The smoother experimental curve is attributed to fabrication tolerances, as well as the effects of the gold layer deposition. Larger deviations in the shape of the diffraction efficiency are observed in the case of TM-polarization and $\varphi=90^{\circ}$. Better agreement is achieved by setting $\varphi$ to $93^{\circ}$ in the simulations, thus indicating the high dependence of the diffraction efficiency on $\varphi$ for TM-polarized radiation. For wavelengths longer than $8.5 \mu \mathrm{m}$, the reflection of the grating in the 0 th $\mathrm{DO}$ approaches the reflectivity of a flat gold surface with $R>97 \%$ for both polarizations. As expected, for longer MIR wavelengths, the incident electric field is unaffected by the periodic grating structure, with the grating behaving as a plane metallic reflector. The minimum

(a)

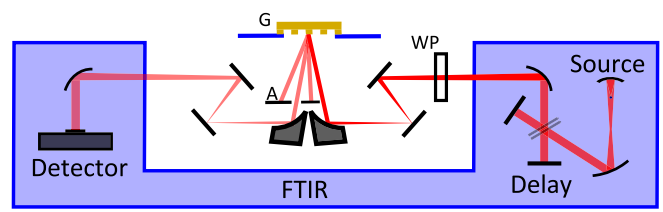

(b)

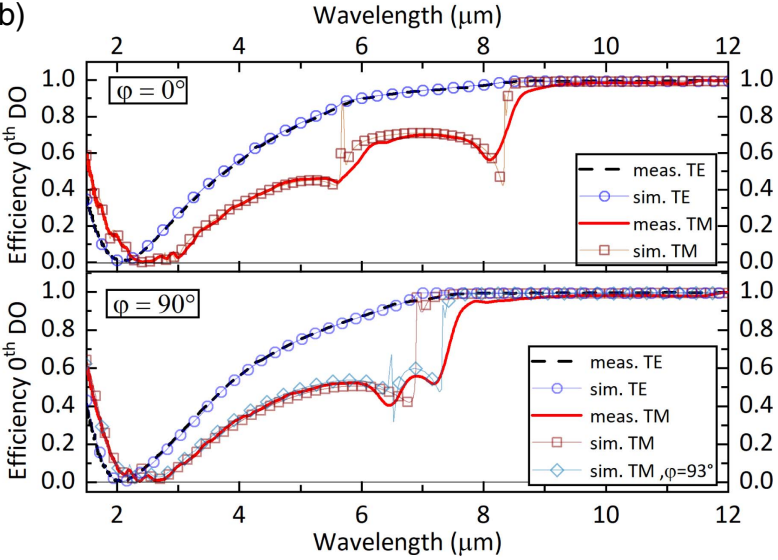

Fig. 2. Diffraction efficiencies simulated and measured. (a) Commercial FTIR for reflection measurements at $10^{\circ}$, modified with a polarizer and iris for the measurement of only the 0th DO. G, grating; WP, wire grid polarizer; A, aperture. (b) Comparison of measured and simulated diffraction efficiencies into the 0 th order. The incident angle is $10^{\circ}$, the grating depth is $0.6 \mu \mathrm{m}$, and the grating period $7 \mu \mathrm{m}$. 
measured efficiency in Fig. 2(b) is around $0.8 \%$ at $2.1 \mu \mathrm{m}$ for TE-polarization and $0.1 \%$ at $2.45 \mu \mathrm{m}$ for TM-polarization, meaning that the suppression of narrowband signals by three orders of magnitude is possible with just a single optic. The $5 \%$ suppression bandwidth is approximately 490 (TE) and $650 \mathrm{~nm}$ (TM).

In the case of IPDFG setups, a broadband, ultrashort driving pulse is employed. In order to provide suitable extinction across the entire driving spectrum, the minima of the efficiency curve can be tuned by adapting the incident angle on the grating: with increasing incidence angle, the diffraction minima shift to shorter wavelengths. This tuning behavior allows for the use of multiple identical gratings in series in order to cover a larger bandwidth. This concept was demonstrated using a high-power $2 \mu \mathrm{m}$ femtosecond laser employed in IPDFG experiments [14], as discussed in the following. The driving spectrum had a $20 \mathrm{~dB}$ bandwidth of around $500 \mathrm{~nm}$, with an average power of $23 \mathrm{~W}$. Using three gratings with different incident angles, a suppression of more than $30 \mathrm{~dB}$ was achieved. Figure 3(a) shows the simulated reflectance of up to three gratings for the spectral region of the driving pulse and for three gratings, as well the measured spectrum after the arrangement. The type- 1 phase matching in the used IPDFG process leads to orthogonal polarizations of the MIR beam and NIR beam. Figure 3(b) shows a transmission of $>85 \%$ in the MIR spectral region above $7 \mu \mathrm{m}$. Due to the grating setup

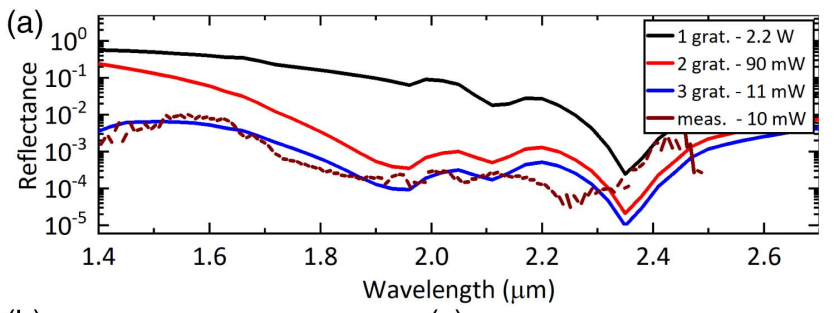

(b)

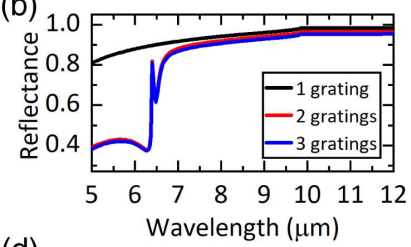

(c)
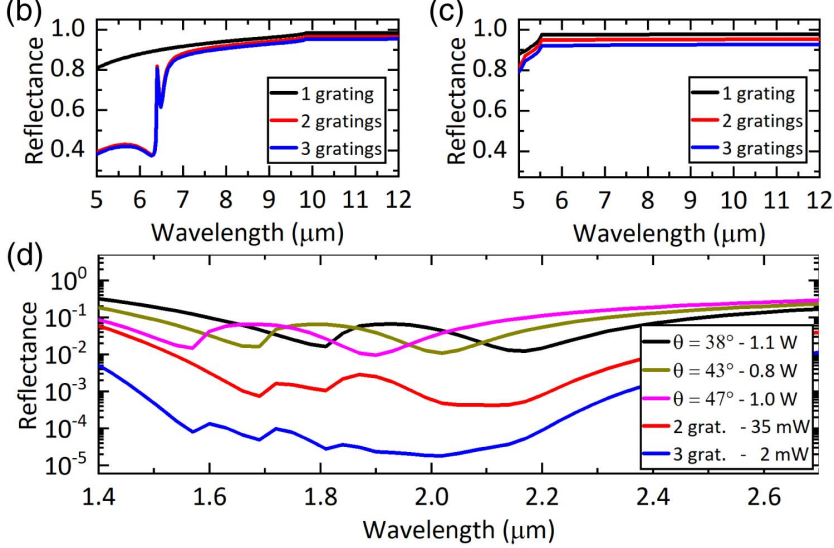

Fig. 3. Suppression of a broadband 23 W NIR driving pulse and reflectance of MIR for different grating settings. (a) Suppression for three implemented gratings $\left(\varphi=90^{\circ}\right.$; TE-pol; $\theta=24^{\circ}, 52^{\circ}$ and $\varphi=0^{\circ}$; TM-pol; $\theta=24^{\circ}$ ) and measured suppression of the NIR spectrum. (b) MIR reflectance for gratings as in (a) with MIR beam polarization orthogonal to that of the NIR beam. (c) MIR beam reflectance for higher efficiencies at shorter wavelengths $\left(\varphi=90^{\circ}\right.$, TEpol $\theta=38^{\circ}, 43^{\circ}, 47^{\circ}$ ). (d) NIR beam suppression by $>40 \mathrm{~dB}$ with grating configuration as in (c) and TM-polarization. The 0th-order diffraction efficiency minima shift to shorter wavelengths with an increasing incidence angle. replacing a dielectric long-pass filter based on a germanium substrate (with which thermal lensing was observed) in the vacuum chamber available for the experiments reported here, the choice of angles was limited. An ideal positioning of the gratings for higher reflectance around $5 \mu \mathrm{m}$ would lead to a MIR reflectance close to that of three gold mirrors (>92\%) for wavelengths longer than $5.5 \mu \mathrm{m}$ and to a suppression of the driving pulse by $40 \mathrm{~dB}$, shown in Figs. 3(c) and 3(d), respectively. The use of incident angles close to $45^{\circ}$ facilitates integration into common optical setups, while sufficient beam separation is achieved after several centimeters. By operating in an all-reflective regime and using silicon substrates the grating affords good thermal stability.

The power scalability was investigated for average powers up to $40 \mathrm{~W}$ (pulse energy: $0.8 \mu \mathrm{J}$, power density: $831 \mathrm{~W} / \mathrm{cm}^{2}$ ). A schematic of the experimental test setup for one grating is shown in Fig. 4(a). The laser used was a femtosecond fiber chirped-pulse amplification system, operating at a central wavelength of $1.96 \mu \mathrm{m}$, with a pulse width of $250 \mathrm{fs}$ [14]. The experiment was performed in vacuum, which is necessary to avoid absorption of the $2 \mu \mathrm{m}$ light by ambient water vapor. For testing, the grating was glued to a kinematic mirror mount with thermal adhesive. While in these experiments, the back side of the grating was not water-cooled, the reflective geometry of the filter allows efficient water cooling of the entire substrate (which is not possible with transmissive optics). In order to characterize the influence of the residual absorption, the beam profile of the 0th DO off the grating was recorded for increasing incident power. To further differentiate whether changes in the beam profile originate from the grating or the laser, a control experiment with the reflection off a dielectric-coated high-reflectivity NIR mirror and glass wedge was performed. Figure 4(b) shows the beam profile reflected from the grating for powers ranging from 1 to $40 \mathrm{~W}$. Figure $4(\mathrm{c})$ presents a

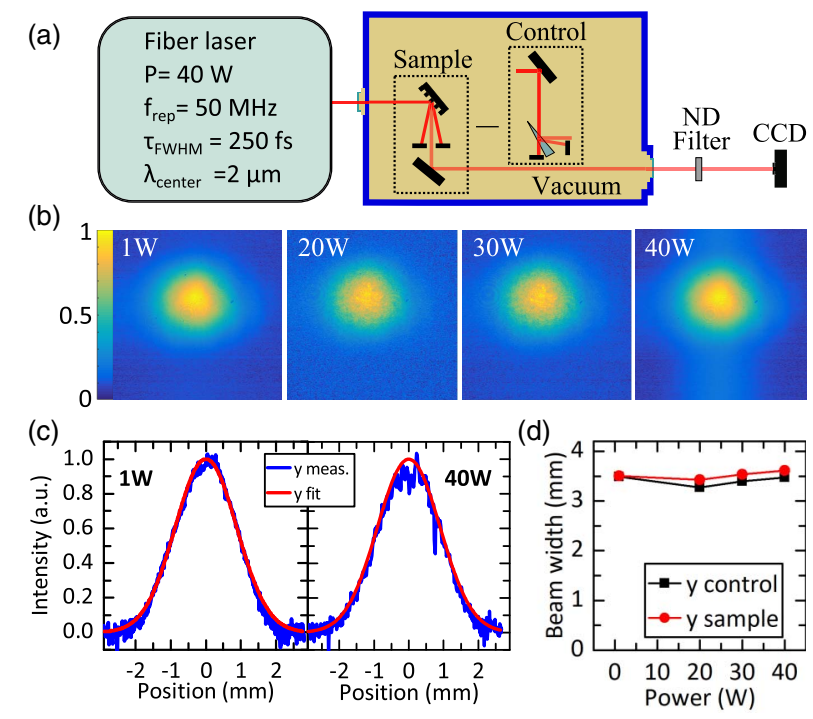

Fig. 4. Thermal stability measurements. (a) Setup for thermal stability test is in vacuum. The control test uses a dielectric mirror and glass wedge to sample the beam. (b) NIR beam profiles of the grating 0th DO at different powers taken with a silicon CCD, employing twophoton absorption. (c) Linecuts along the y-axis measured and fit with a Gaussian function. (d) $1 / e^{2}$ beam diameter of the $y$ axis Gaussian fit at different powers for the sample and control setup. 
vertical linecut through the maximum of the beam profile, along with a Gaussian fit, used to minimize the effect of noise from the two-photon imaging sensor. Figures 4(b) and 4(c) show no substantial changes with increasing incident power, which would be expected if there was any significant thermal gradient present on the grating surface. This is also underlined in Fig. 4(d), which shows the measured beam width versus the incident power. Only a minor difference in the beam width betweeen control and sample is seen, thus indicating that the thermal load contribution on the grating is small. In this test, the maximum fluence is $4.2 \mu \mathrm{J} / \mathrm{cm}^{2}$, well below the typical ablation thresholds of thin gold films (typically in the order of $0.1 \mathrm{~J} / \mathrm{cm}^{2}[26]$ ). Therefore, the power scalability is mostly limited by thermal effects, e.g., deformation or melting. At increased power levels $(>40 \mathrm{~W})$, these effects could be mitigated by larger spot sizes on the gratings.

In conclusion, we have reported the design, fabrication, and experimental characterization of a high-power-suitable and chromatic-dispersion-free long-pass filter based on a diffractiongrating geometry. The gold-coated silicon gratings are capable of separating high-power, broadband NIR light from a collinearly propagating MIR beam by taking advantage of the differing diffraction efficiency between the short and long wavelength components. The used gratings have been shown to be suitable for idler wavelengths above $5.5 \mu \mathrm{m}$, with the reflectivity limited only by the intrinsic gold absorption and, thus, rendering these gratings suitable for a wide range of MIR and $\mathrm{THz}$ applications.

Numerical simulations of the grating structure and incident beam geometry have been shown to accurately predict the filter characteristics, allowing the necessary optimization of the grating parameters and placement of the gratings for a tailored blocking range of the driving pulse and a suitable MIR diffraction. Although the slope is not as steep as possibly achievable in multilayer optics, it is sufficient for IPDFG applications with its usually distinct spectrally separated pump and idler wavelength ranges. By using a series of filters, broad bandwidth driving pulses can be suppressed by several orders of magnitude, while simultaneously keeping a high throughput of MIR light and not introducing any additional dispersion to ultrashort MIR pulses. The power scaling of the gratings has been investigated, with no significant thermal effect observed with incident average powers of up to $40 \mathrm{~W}$. Most recently, these optics were successfully applied in the generation of single-cycle transients in the molecular fingerprint region via IPDFG [14], corroborating their suitability for minimizing the dispersion of the MIR beam path in a time-domain experiment. They are likely to find application in experiments with even longer wavelengths such as femtosecond-laser-driven terahertz spectroscopy (see, e.g., $[27,28]$ and references therein).

Funding. Munich-Centre for Advanced Photonics (MAP); Deutsche Forschungsgemeinschaft (DFG) (KR4768/1-1).

Acknowledgment. The authors are grateful to the group of Prof. Limpert for support with the fiber lasers and Prof. Krausz and Prof. Tünnermann for helpful discussions.

\section{REFERENCES}

1. W. Demtröder, Molecular Physics: Theoretical Principles and Experimental Methods (Wiley, 2008).

2. A. Barth and P. I. Haris, Biological and Biomedical Infrared Spectroscopy (IOS Press, 2009).

3. J. Haas and B. Mizaikoff, Annu. Rev. Anal. Chem. 9, 45 (2016).

4. K. Fritsch, M. Poetzlberger, V. Pervak, J. Brons, and O. Pronin, Opt. Lett. 43, 4643 (2018)

5. T. Saule, S. Holzberger, O. de Vries, M. Plötner, J. Limpert, A. Tünnermann, and I. Pupeza, Appl. Phys. B 123, 6877 (2017).

6. C. Gaida, T. Heuermann, M. Gebhardt, E. Shestaev, T. P. Butler, D. Gerz, N. Lilienfein, P. Sulzer, M. Fischer, R. Holzwarth, A. Leitenstorfer, I. Pupeza, and J. Limpert, Opt. Lett. 43, 5178 (2018).

7. J. Zhang, K. F. Mak, and O. Pronin, IEEE J. Sel. Top. Quantum Electron. 24, 1 (2018).

8. A. Schliesser, N. Picqué, and T. W. Hänsch, Nat. Photonics 6, 440 (2012).

9. A. A. Lanin, A. A. Voronin, A. B. Fedotov, and A. M. Zheltikov, Sci. Rep. 4, 6670 (2014).

10. M. Seidel, X. Xiao, S. A. Hussain, G. Arisholm, A. Hartung, K. T. Zawilski, P. G. Schunemann, F. Habel, M. Trubetskov, V. Pervak, O. Pronin, and F. Krausz, Sci. Adv. 4, eaaq1526 (2018).

11. I. Pupeza, D. Sánchez, J. Zhang, N. Lilienfein, M. Seidel, N. Karpowicz, T. Paasch-Colberg, I. Znakovskaya, M. Pescher, W. Schweinberger, V. Pervak, E. Fill, O. Pronin, Z. Wei, F. Krausz, A. Apolonski, and J. Biegert, Nat. Photonics 9, 721 (2015).

12. C. Gaida, M. Gebhardt, T. Heuermann, F. Stutzki, C. Jauregui, J. Antonio-Lopez, A. Schülzgen, R. Amezcua-Correa, A. Tünnermann, I. Pupeza, and J. Limpert, Light: Sci. Appl. 7, 94 (2018).

13. J. Zhang, K. F. Mak, N. Nagl, M. Seidel, D. Bauer, D. Sutter, V. Pervak, F. Krausz, and O. Pronin, Light: Sci. Appl. 7, 17180 (2017).

14. T. P. Butler, D. Gerz, C. Hofer, J. Xu, C. Gaida, T. Heuermann, M. Gebhardt, L. Vamos, W. Schweinberger, J. A. Gessner, T. Siefke, M. Heusinger, U. Zeitner, A. Apolonski, N. Karpowicz, J. Limpert, F. Krausz, and I. Pupeza, Opt. Lett. 44, 1730 (2019).

15. I. Pupeza, M. Huber, W. Schweinberger, M. Trubetskov, S. A. Hussain, L. Vamos, O. Pronin, F. Habel, V. Pervak, N. Karpowicz, E. Fill, A. Apolonski, M. Zigman, A. M. Azzeer, and F. Krausz, Conference on Lasers and Electro-Optics (CLEO)/Europe \& EQEC (2017), p. 1

16. A. Kowligy, H. Timmers, A. Lind, U. Elu, F. Cruz, P. G. Schunemann, J. Biegert, and S. A. Diddams, CLEO: Applications and Technology (2018), p. JTh5B.6.

17. G. J. Hawkins, R. Hunneman, M. T. Gardner, and G. T. Babcock, Infrared Phys. Technol. 39, 297 (1998).

18. T. Amotchkina, M. Trubetskov, M. Schulz, and V. Pervak, Opt. Express 27, 5557 (2019).

19. F. Habel and V. Pervak, Appl. Opt. 56, C71 (2017).

20. J. U. White, J. Opt. Soc. Am. A 37, 713 (1947).

21. D. C. Yost, T. R. Schibli, and J. Ye, Opt. Lett. 33, 1099 (2008).

22. M. Trost, S. Schröder, A. Duparré, S. Risse, T. Feigl, U. D. Zeitner, and A. Tünnermann, Opt. Express 21, 27852 (2013).

23. U. D. Zeitner, M. Oliva, F. Fuchs, D. Michaelis, T. Benkenstein, T. Harzendorf, and E.-B. Kley, Appl. Phys. A 109, 789 (2012).

24. K. C. Johnson, "GD-Calc," 2017, http://kjinnovation.com/GD-Calc .html.

25. A. Ciesielski, L. Skowronski, M. Trzcinski, E. Górecka, P. Trautman, and T. Szoplik, Surf. Sci. 674, 73 (2018).

26. N. Bonod and J. Neauport, Adv. Opt. Photonics 8, 156 (2016).

27. E. Matsubara, M. Nagai, and M. Ashida, Appl. Phys. Lett. 101, 11105 (2012).

28. J. Xu, B. Globisch, C. Hofer, N. Lilienfein, T. Butler, N. Karpowicz, and I. Pupeza, J. Phys. B 51, 154002 (2018). 